\title{
Contribuições aos estudos anatômicos de Bromeliaceae (Poales) sob uma perspectiva filogenética
}

\section{Fernanda Maria Cordeiro de Oliveira}

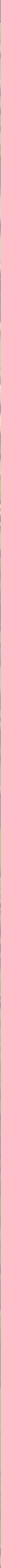



Fernanda Maria Cordeiro de Oliveira

Contribuições aos estudos anatômicos de Bromeliaceae (Poales) sob uma perspectiva filogenética

Contribution to the anatomical studies of Bromeliaceae (Poales) under a phylogenetic perspective 



\section{Fernanda Maria Cordeiro de Oliveira}

Contribuições aos estudos anatômicos de Bromeliaceae (Poales) sob uma perspectiva filogenética

Contribution to the anatomical studies of Bromeliaceae (Poales) under a phylogenetic perspective

Tese apresentada ao Instituto de Biociências da Universidade de São Paulo, para a obtenção de Título de Doutora em Ciências Biológicas, na Área de Botânica.

Orientadora: Profa. Dra. Gladys Flavia de Albuquerque Melo de Pinna

Co-Orientadora: Profa. Dra. Maria das Graças Wanderley. 
Oliveira, Fernanda Maria Cordeiro

Contribuições aos estudos anatômicos de Bromeliaceae (Poales) sob uma perspectiva filogenética

159 páginas

Tese (Doutorado) - Instituto de Biociências da Universidade de São Paulo. Departamento de Botânica.

1. Reconstrução de caracteres ancestrais; 2. Evolução; 3. Complexo Nidularióide; 4. Anatomia floral; 5. Tricomas glandulares I Universidade de São Paulo. Instituto de Biociências. Departamento de Botânica.

\section{Comissão Julgadora}

$\operatorname{Prof}(a) . \operatorname{Dr}(a)$.

$\operatorname{Prof}(a) . \operatorname{Dr}(a)$.

$\operatorname{Prof}(\mathrm{a}) . \operatorname{Dr}(\mathrm{a})$

$\operatorname{Prof}(\mathrm{a}) . \operatorname{Dr}(\mathrm{a})$

Profa. Dra. Gladys Flavia de Albuquerque Melo de Pinna (Orientadora) 
Àquela que me amou desde o primeiro olhar. 

"A humanidade é parte de um vasto universo em evolução. A Terra, nosso lar, está viva com uma comunidade de vida única. As forças da natureza fazem da existência uma aventura exigente e incerta, mas a Terra providenciou as condições essenciais para a evolução da vida. (...) O meio ambiente global com seus recursos finitos é uma preocupação comum de todas as pessoas. A proteção da vitalidade, diversidade e beleza da Terra é un dever sagrado." Carta da Terra (1992) 



\section{Agradecimentos}

Quatro anos podem passar muito devagar quando não se gosta do que faz. Mas quando você gosta, passam rápido... Para mim, no final das contas, quatro anos passaram voando e deixam ótimas lembranças. E, se passou rápido, foi devido a todo o apoio que recebi, desde a minha chegada em São Paulo até agora.

Agradeço primeiramente ao Instituto de Biociências da Universidade de São Paulo (IB-USP), instituição que me recebeu e forneceu toda a infraestrutura para a realização deste trabalho e também a todos os professores e funcionários.

Ao CNPq, pela bolsa concedida, que possibilitou que este trabalho fosse realizado.

Agradeço com muito carinho, minha querida orientadora, Professora Dra. Gladys Flavia de Albuquerque Melo-de-Pinna. Agradeço por todos os momentos de orientação, pela confiança desde o primeiro dia, quando este projeto ainda era um sonho. Pelos ensinamentosde botânica e de vida. Agradeço também por todos os momentos deamizade, todas as conversas e conselhos!

À professora Dra. Maria das Graças Lapa Wanderley, que tão prontamente aceitou me coorientar. Obrigada pelas discussões sobre morfologia e pela colaboração com o doutorado.

Ao professor Dr. Rafael Batista Louzada, que tão bem me acolheu em seu laboratório em Recife. Obrigada pela ajuda com o mapeamento dos caracteres, pelos almoços com muitas discussões sobre Bromeliaceae e pela colaboração neste trabalho! Não posso deixar de agradecer aqui, também, à Dra. Maria Claudia Medeiros pela ajuda com o R e por todos os bons momentos em Recife. 
Agradeço aos membros que fizeram parte da minha banca de qualificação: Profa. Dra. Nanuza Luiza de Menezes, Profa. Dra. Vera Lúcia Scatena e Prof. Dr. Benoît Loeille. Obrigada por todos os conselhos e recomendações referentes ao projeto desta tese.

Às técnicas do Laboratório de Anatomia Vegetal, Gilese Costa e Tássia dos Santos, por toda a ajuda que recebi desde o meu primeiro dia na USP. Obrigada pelas dicas sobre as técnicas, pelos conselhos, pela ajuda com a parte prática. Obrigada por todas as vezes em que se dispuseram a me ajudar e que me incentivaram quando as coisas não davam certo. Por todos os “tenta outra vez Fê, vai dar certo!”. E, claro, por todos os momentos na copinha para o café...

Aos professores do Laboratório de Anatomia Vegetal da USP: Profa. Dra. Nanuza Luiza de Menezes, Prof. Dr. Diego Demarco, Prof. Dr. Gregório Ceccantini, Profa. Dra. Gladys Flavia de Albuquerque Melo-de-Pinna, Profa. Dra. Verônica Angyalossy e Dra. Berta Lange de Morretes. Obrigada por todos os ensinamentos botânicos, pelas disciplinas ministradas, pelas referências, artigos, livros. Agradeço também por todas as vezes em que tive dúvidas e vocês estavam sempre prontos a saná-las.

Aos meus queridos colegas do Laboratório de Anatomia Vegetal. Seria impossível nomear todos, mas vocês fazem parte desta história.

Aos professores do Laboratório de Morfo-Taxonomia Vegetal da UFPE, Prof. Dr. Rafael Batista Louzada, Prof. Dr. Benoît Loeille e Prof. Dr. Marccus Alves. Obrigada por me permitirem fazer parte deste laboratório, por me apresentarem a Caatinga!

Aos meus queridos irmãos de orientação, à equipe Melo-de-Pinna. Obrigada Aline Ogura, Bruno Edson, Carlos Valério, José Hernandes, Juliana Brasileiro, Leyde Nunes, Rafael Cruz, Renata Lemos, Ricardo Vita pela colaboração no laboratório, pelas sugestões e por todo o carinho. 
Aos colegas do Laboratório de Morfo-Taxonomia Vegetal: Beta Ferralc, Beth Córdula, Camila Alcantara, Danielly Lucena, Débora Cavalcanti, Edlley Pessoa, Marcio Lucas, Marily Jhullis, Naédja Kaliéri, Regina Carvalho e Thales Coutinho.

Ao Des. Elton Leme, que abriu as portas do seu refúgio dos Gravatás em Teresópolis, permitindo que eu coletasse o material necessário para o desenvolvimento do primeiro capítulo desta tese.

Agradeço em especial ao Rafael Cruz, por toda a paciência em me ensinar a usar o mesquite! Por me ajudar com os alinhamentos e com o "terrível” Mr. Bayes. Também por todos os momentos agradáveis e de descontração.

À Aline Ogura, por todos os cafézinhos, onde a gente colocava a conversaem dia. Obrigada por todo o carinho!

À Renata Lemos -Renatiiiiinhaaaa! Obrigada por estar "juntinho" todos os momentos! Obrigada pela paciência, por todas as jantinhas maravilhosas, por me escutar e me aconselhar. Obrigada pela amizade, sempre!

Aos amigos de SP, nossa galerinha reunida de todos os meses! Obrigada Bruno Sales, Gustavo Burin, José Hernandes, Tássia Santos e Renata Lemos por fazerem de São Paulo uma cidade tão acolhedora! Obrigada por todas as gargalhadas até o ar me faltar, pelas discussões de política, pelos filmes, pelas pipocas, pelas gordices! Obrigada por estarem sempre pertinho!

Às minhas queridas Viseiras: Carolina Bastos, Luíza Teixeira, Mariana Victório, Yasmin Hirao e Thaíla Vieira. Obrigada por todos os momentos de risos, todos os encontros e obrigada pela motivação sempre! 
Aos queridos Karla Figueiredo e Mário Lima que me receberam em sua casa no período em que estive em Recife. Obrigada por me fazerem me apaixonar pelo Nordeste e por todo o carinho que me deram.

À minha família, que me apoiou incondicionalmente para que esse sonho se tornasse realidade. Minha Mãe Olga Oliveira, Irmão Darley Oliveira e minha pequena Gabriela Oliveira! Obrigada por todas as ligações, por todas as vezes que me buscaram na rodoviária, todos os momentos de carinho!

E ao Weslley Dalcol, que me apoiou desde o início. Obrigada por compreender os momentos de ausência! 


\section{Resumo}

Bromeliaceae ocupa posição basal na Ordem Poales e é considerada monofilética, tendo sinapomorfias morfológicas e moleculares. Atualmente é subdividida em oito subfamílias, sendo Bromelioideae a subfamília com maiores problemas na delimitação genérica. Nesse contexto encontra-se o Complexo Nidularióide, formado pelos gêneros Nidularium Lem., Wittrockia Lindm., Neoregelia L.B.Sm., Canistropsis (Mez) Leme e Edmundoa Leme. A dificuldade na delimitação destes gêneros se dá pelo uso de um grande número de caracteres não exclusivos, que mostram o íntimo relacionamento entre estes gêneros. Embora estes gêneros não sejam monofiléticos, como indicam as filogenias recentes, o Complexo Nidularióide como um todo sempre emerge em um clado, indicando que formam uma unidade taxonômica. Neste contexto, analisamos caracteres morfológicos e anatômicos a fim de estabelecer novas sinapomorfias para o Complexo. Nossos resultados indicam que os principais caracteres morfológicos utilizados na delimitação dos gêneros do complexo Nidularióide são homoplásticos. A maior parte dos caracteres anatômicos também representam homoplasias. No entanto a anatomia foliar mostrou-se útil, provendo novas sinapomorfias para o grupo, tais como a presença de células da ala alongadas nos tricomas, e presença de células epidérmicas adaxiais com paredes levemente espessadas na lâmina foliar. No segundo capítulo, analisamos a anatomia floral de 16 espécies de Bromeliaceae, pertencente a três das oito subfamílias, a fim de estabelecer caracteres anatômicos florais úteis na sistemática do grupo, bem como discutilos sob uma perspectiva ecológica e filogenética. Uma nova sinapomorfia é proposta para Pitcairnoideae a partir de dados sobre a vascularização do gineceu. No terceiro capítulo, analisamos o desenvolvimento foliar de três espécies de Tillandsia L. (Tillandsoideae). Neste estudo, foi possível registrar a presença de coléteres nas bainhas foliares próximas aos ápices caulinares de T. tricholepis (L.) L. Estas estruturas são responsáveis pela proteção do meristema apical caulinar (MAC) por meio de seu exsudado. Este estudo é o primeiro registro destas estruturas em Bromeliaceae. 


\begin{abstract}
Bromeliaceae ocupies a basal position in Poales Order and is considered monophyletic by morphological and molecular sinapomorphies. The family is currently subdivided into eight subfamilies. Bromelioideae has major problems in generic delimitation. In this context is included Nidularioid Complex, formed by the genera Nidularium Lem., Wittrockia Lindm., Neoregelia L.B.Sm., Canistropsis (Mez) Leme e Edmundoa Leme. The difficulty to generic delimitation in these genera occurs by the presence of a great number of non-exclusive character, which demonstrates their intimate relashioship. Although recent phylogenies indicate these genera are not monophyletic, Nidularioid Complex always emerges as a clade, which suggests they are a true taxonomic entity. In this context, we analyzed morphological and anatomical characters to establish new sinapomorphies for the Complex. Our results indicates that the main morphological characters used in genera delimitation on Nidularioid Complex are homoplastic. The major part of anatomical characters are also homoplastic. However, leaf anatomy shows to be useful, providing new synapomorphies for this group, such as the presence of trichomes with elongated wing's cells and the presence of adaxial epidermal cells with lightly thickened walls on leaf blade. On the second chapter, we analyzed floral anatomy of 16 species of Bromeliaceae, belonging to three out of eight subfamilies, to establish anatomical floral characters useful in the group sistematics, as well as discuss it under an ecological and phylogenetic perspective. A new synapomorphy for Pitcairnoideae is established, by data of gynoicea vascularization. On the third chapter, we analyzed leaf development of three species of Tillandisia L. (Tillandsoideae). In this study, it was possible to describe the presence of colleters in leaf sheath, next to shoot apices of T. tricholepis (L.) L. These structures are responsible by shoot apical meristem's (SAM) protection by their secretion. This is the first record of colleters in Bromeliaceae.
\end{abstract}




\section{Índice}

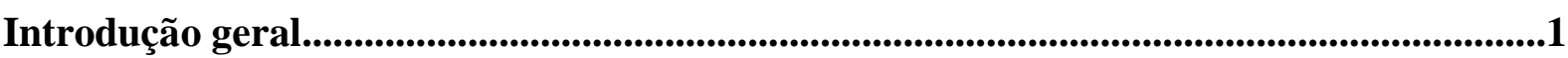

1.1 Caracterização morfológica de Bromeliaceae.........................................................3

1.2 Aspectos anatômicos em Bromeliaceae.................................................................4

1.3 Importância econômica e ecológica de Bromeliaceae.............................................8

1.4 Estrutura Geral da Tese..............................................................................10

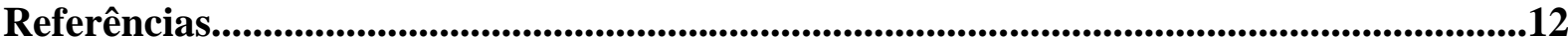

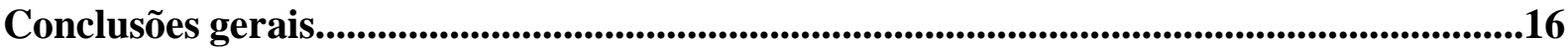


Introdução Geral 



\section{Introdução Geral}

Bromeliaceae possui cerca de 3.350 espécies, distribuídas em 58 gêneros (Luther, 2012), sendo a sua distribuição predominantemente neotropical (Smith \& Downs, 1974). A exceção é Pitcairnia feliciana (A. Chev.) Harms \& Mildbr. que ocorre na costa oeste da África, resultado de uma dispersão a longa distância (Jacques-Felix, 2000; Givnish et al., 2004). A família possui dois grandes centros de diversidade: o Escudo das Guianas e a Costa Leste do Brasil (Givnish et al. 2007). Para o Brasil, são citados 40 gêneros ca. de 1300 espécies, sendo que ca. de 1100 espécies são consideradas endêmicas (BFG, 2015) e alguns táxons são encontrados exclusivamente na Mata Atlântica (Martinelli et al., 2008).

Durante muito tempo, graças a características singulares e de fácil reconhecimento, Bromeliaceae foi considerada a única família pertencente à ordem Bromeliales (Cronquist, 1981; Dahlgren et al. 1985; Gilmartin \& Brown, 1987). Atualmente incluída na ordem Poales, a família tem como grupo-irmão Typhaceae, ocupando a posição basal da ordem (APG IV, 2016). Seu monofiletismo é sustentando tanto por caracteres moleculares - sequência de $n d h \mathrm{~F}$ (Givnish et al. 2007; Givnish et al., 2011), morfológicos - presença de tricomas peltados absorventes (Benzing, 2000), presença de estigmas tipo espiral conduplicado (Brown \& Gilmartin, 1989a) e citogenéticos - número cromossômico básico x=25 (Brown \& Gilmartin, 1989b).

É tradicionalmente dividida em três subfamílias: Bromelioideae, Tillandsoideae e Pitcairnoideae s.l., cujas circunscrições levam em conta principalmente a morfologia foliar, posição do ovário, tipo de fruto e de semente (Smith \& Downs, 1974, 1977, 1979). No entanto, por meio de estudos moleculares, verificou-se que Pitcairnoideae não representava um grupo monofilético. Assim, a família foi então reorganizada em oito subfamílias, a saber: Bromelioideae, Tillandsoideae, Pitcairnoideae s.s., Navioideae, Puyoideae, Brocchinioideae, Hechtioideae e Lindmanioideae (Fig. 1) (Givnish et al. 2007; Givnish et al. 2011). 


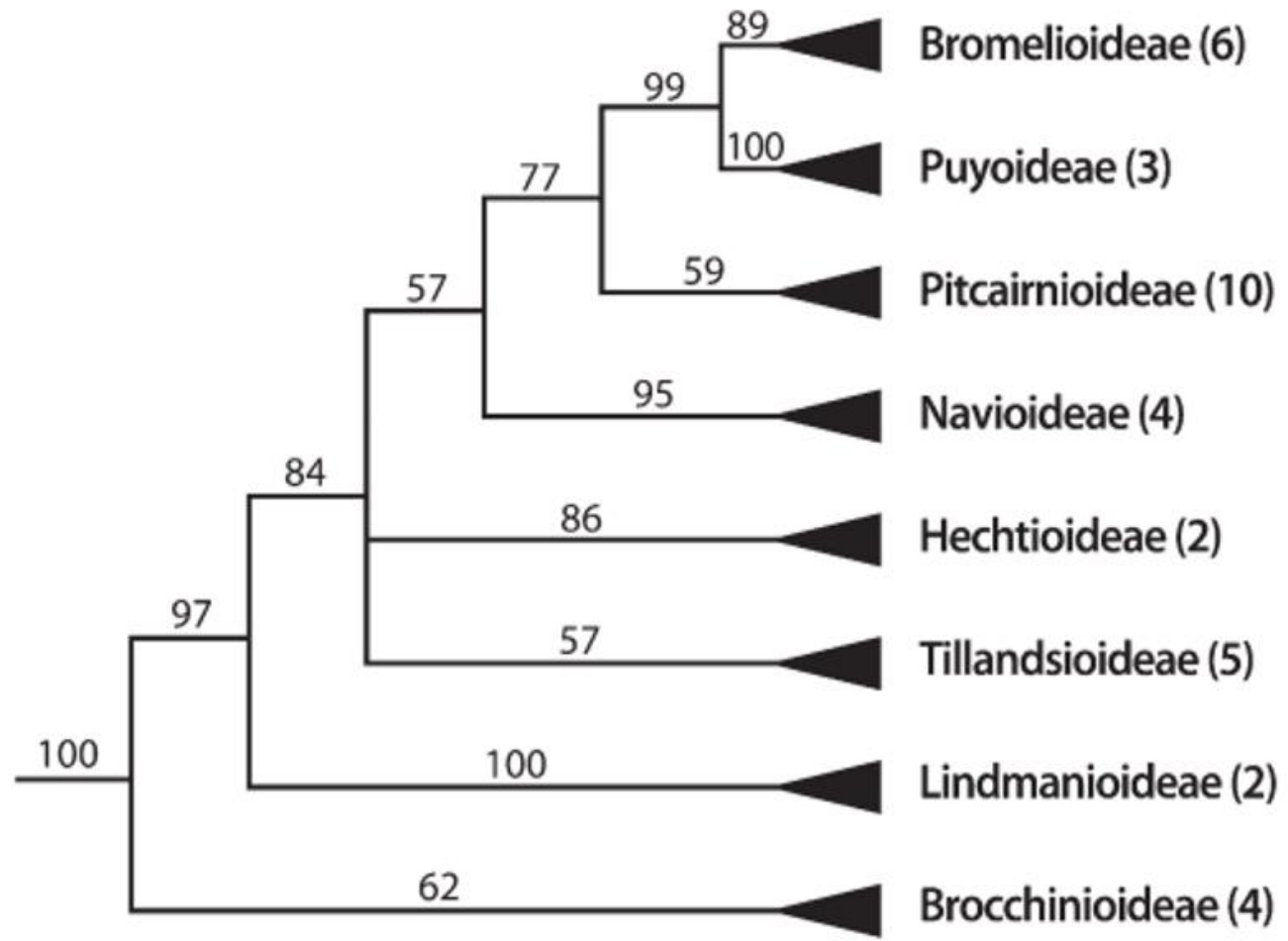

Figura 1: Árvore de máxima parcimônia de consenso stricto, baseada na variação nas sequências plastidiais ndhF, com as relações propostas entre as subfamílias de Bromeliaceae. Grupos externos de sete famílias de Poales não são mostrados. Números acima dos ramos são os valores de bootstrap; números em parênteses após os nomes das subfamílias indicam o número de táxons utilizados nas análises. Retirado de Givnish et al. (2011).

Apesar dos avanços obtidos com o advento da sistemática molecular, muito ainda há para ser feito em relação à sistemática de Bromeliaceae. Segundo Escobedo-Sarti et al. (2013), dos 58 gêneros descritos em Bromeliaceae, apenas 14 são considerados monofiléticos, a saber: Brocchinia Schult. f., Alcantarea Harms, Catopsis Griseb., Glomeropitcairnia Mez, Guzmania Ruiz \& Pav., Werauhia J. R. Grant, Hechtia Klotzsch, Steyerbromelia L. B. Sm., Fosterella L. B. Sm., Puya Molina, Ananas Mill., Araeococcus Brongn., Bromelia L., e Acanthostachys Link, Klotzsch \& Otto. Estes gêneros têm em comum poucas espécies e fácil reconhecimento morfológico. 
Segundo Wanderley \& Moreira (2000) a questão conceitual genérica desta família como um todo tem se mostrado muito complexa. Assim, as autoras recomendam o uso de estudos mais aprofundados nestes grupos, a fim de não tornar a taxonomia da família ainda mais artificial e complexa. As autoras ressaltam ainda a necessidade de uma revisão genérica, utilizando-se de caracteres morfológicos e estudos de populações em seu ambiente natural.

Dentre as subfamílias supracitadas, Bromelioideae é detentora da maior diversidade morfológica refletindo na inclusão de mais da metade dos gêneros de Bromeliaceae (Benzing, 2000). É caracterizada por possuir a margem foliar espinescente, ovário ínfero e fruto tipo baga (Smith \& Downs, 1979). Nesta subfamília é notória a precariedade de delimitação de vários gêneros, sendo que, muitas vezes, apesar de se ter a descrição completa de uma espécie, é difícil decidir a qual gênero pertence (Smith \& Downs, 1979).

Algumas características chaves, tais como a presença de tricomas peltados absorventes ao longo da folha, metabolismo Ácido das Crassuláceas (CAM), e de um tanque formado pela sobreposição das bainhas foliares que acumula água, permitiram a família ocupar variados habitats, ocorrendo desde o nível do mar até altas altitudes (ca. de 4000 metros), em ambientes mésicos e xéricos (Smith \& Down, 1974; Benzing, 1976; Benzing, 2000; Silvestro et al., 2014).

\subsection{Caracterização morfológica de Bromeliaceae}

Bromeliaceae é formada por plantas herbáceas perenes, terrestres, epífitas ou rupícolas, com caule curto ou longo, rizomatoso ou estolonífero com raízes reduzidas absortivas nas plantas terrestres, fixadoras nas epífitas e rupícolas ou ausentes. As folhas são simples, alternas, polísticas à dísticas, recobertas por tricomas peltados absorventes. Por vezes o imbricamento da bainha foliar forma uma cisterna que acumula água, minerais e detritos orgânicos. Bainha foliar geralmente aberta, pouco ou muito distinta da lâmina foliar. Lâmina foliar pode apresentar margem lisa ou espinescente. As inflorescências são terminais indeterminadas, simples ou compostas. Flores solitárias são raras, como em Tillandsia usneoides L. As flores 
são bissexuadas com perianto diferenciado em cálice e corola, dispostas na axila de brácteas, frequentemente coloridas. As sépalas podem ser livres ou conadas. As pétalas, livres ou conadas, muitas vezes apresentam um par de apêndices em sua base e algumas vezes calosidades longitudinais. $\mathrm{O}$ androceu é formado por duas séries de três estames cada, filetes livres ou conados, as vezes adnatos às pétalas. O ovário pode ser súpero, ínfero ou semi-ínfero, tricarpelar e trilocular, com placentação axial, apresentando nectários septais; o estigma é trífido, podendo ser classificado em simples-ereto, espiral-conduplicado ou laminar-convuluto. O fruto pode ser do tipo cápsula septícida ou baga; sementes sem apêndices, aladas ou plumosas (Smith \& Downs, 1974; Cronquist,1981; Wanderley \& Martins, 2007).

\subsection{Aspectos anatômicos em Bromeliaceae}

Sem dúvida alguma os tricomas peltados absorventes, frequentemente chamados de escamas, são as estruturas anatômicas mais estudadas na família (Benzing, 2000). Diversos pesquisadores buscaram compreender sua organização anatômica e funcionamento (Mez, 1896; Benzing, 1976; Tomlinson, 1969). Estes tricomas, na ausência de raízes (como em algumas espécies de Tillansia spp.), ou quando estas estão presentes mas têm função de fixação no substrato (como em espécies saxícolas ou epífitas), são responsáveis pela absorção de água de nutrientes. Atualmente, sabe-se que a presença de tricomas peltados em Bromeliaceae é uma das características que permitiram a adaptação radiativa da família (Benzing, 2000).

Estes tricomas são compostos, geralmente, de uma célula basal, um pedicelo (que pode ter um número variado de células), célula domo, disco e ala. As células do disco, também chamadas de centrais, conectam a ala ao pedicelo (Krauss, 1949; Tomlinsom, 1969) (Fig. 2 A$\mathrm{B}, 3 \mathrm{~A})$.

Sendo a maior parte da família epífita ou saxícola, as Bromeliaceae sofrem estresse hídrico, aproveitando-se da umidade do ar ou de água acumulada em suas cisternas, também 
chamadas de fitotelma (Benzing, 2000). A rápida absorção de água dá-se por meio de um mecanismo complexo, com envolvimento ativo das escamas presentes nas folhas e foi descrito por Benzing (1976). Além da absorção de água por meio dos tricomas, e da presença de uma cisterna que formada pelo imbricamento de suas folhas, as Bromeliaceae, em geral, contam com um reservatório interno de água proporcionado pela suculência de suas folhas. Tal suculência é dada pela presença de um tecido armazenador de água, denominado hipoderme aquífera (Tomlinson, 1969).

Outra característica da família é a presença de células epidérmicas, nas folhas, com paredes anticlinais sinuosas em vista frontal e contendo um cristal esférico de sílica (Krauss,1949; Tomlinsom, 1969) (Fig. 2D, Fig. 3B). Muito já foi discutido sobre a função de tais cristais, e acredita-se que estejam envolvidos na reflexão da luz (Prychid et al. 2004).

As folhas de Bromeliaceae possuem, ainda, canais de aeração longitudinais preenchidos por células do parênquima braciforme, intercalados com os feixes vasculares Tais canais têm comunicação direta com as câmaras subestomáticas. (Krauss, 1949; Tomlinson, 1969; Benzing, 2000) (Fig. 2C, Fig. 3C-D). 

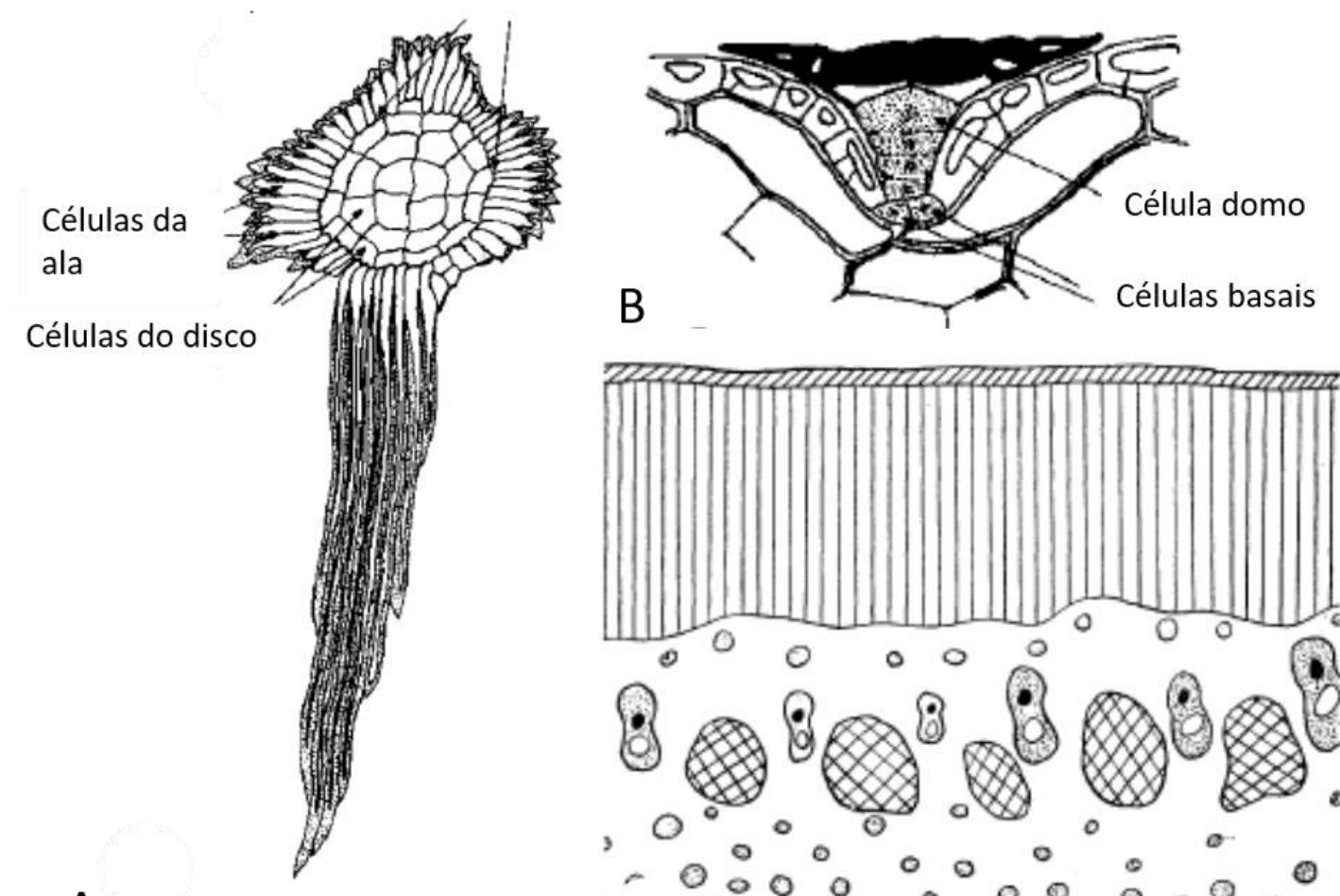

A
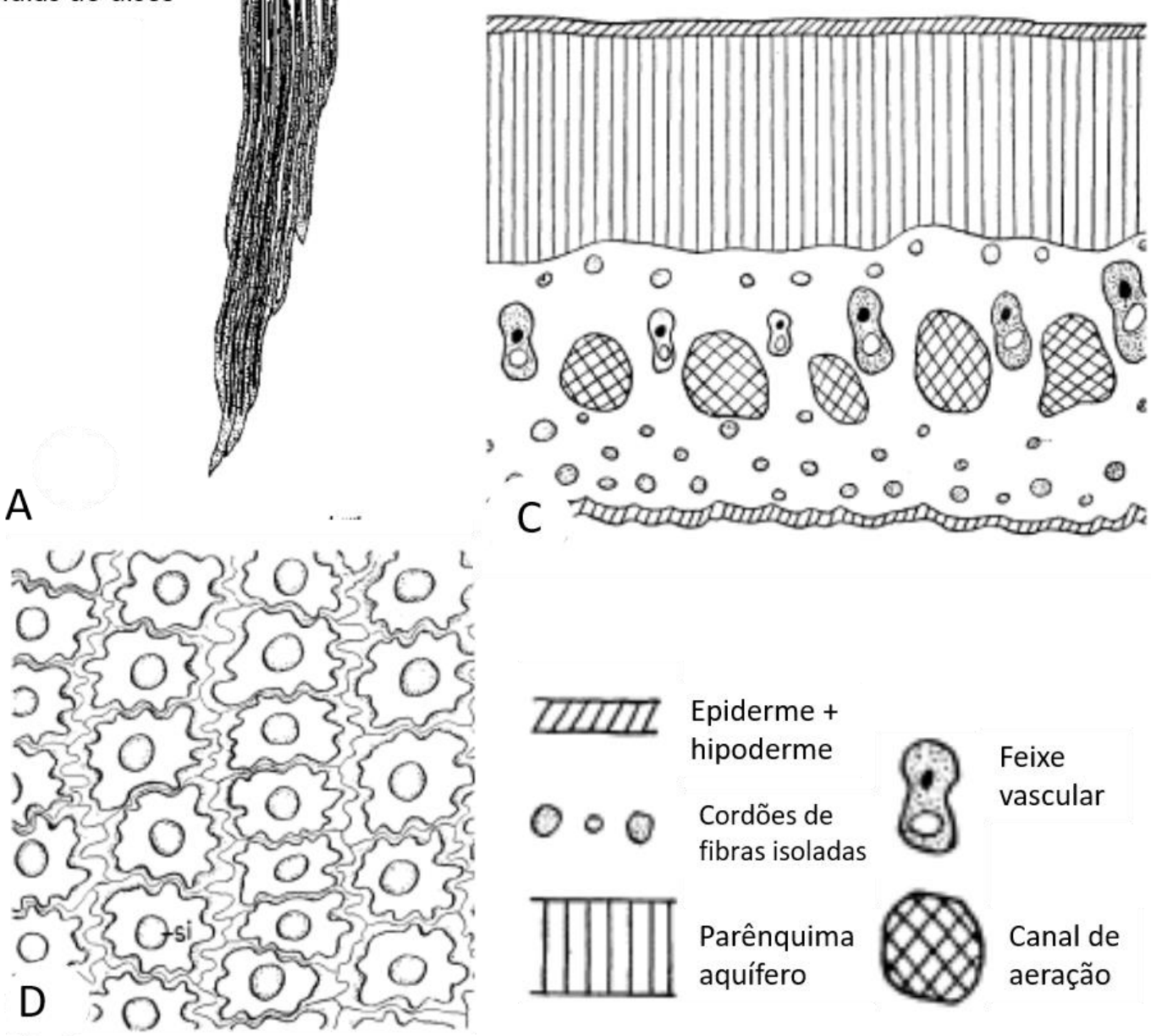

Figura 2: Características anatômicas foliares de Bromeliaceae. A: Tricoma peltado absorvente em visão frontal. B: Tricoma peltado em secção transversal. C: Secção transversal da lâmina foliar de Ananas comusus L. D: Epiderme adaxial em vista frontal de A. comusus. A-B: Modificado de Benzing (2000), redesenhado de Tomlinson (1969). C-D: Modificado de Krauss (1949). 

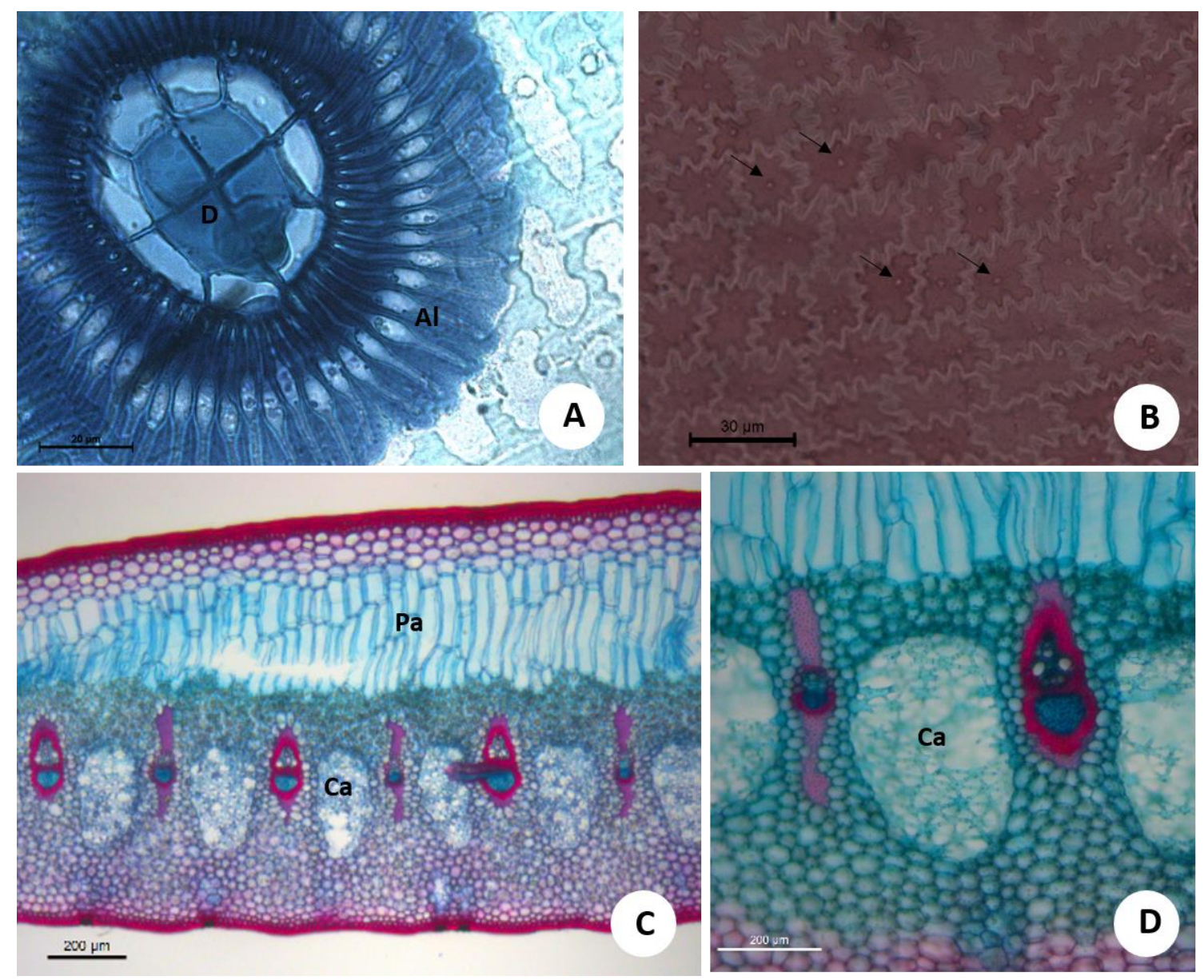

Figura 3: Características anatômicas das folhas de Bromeliaceae. A: Tricoma peltado de Vriesea platynema Gaudich. em vista frontal B: Epiderme adaxial da bainha foliar de Neoregelia compacta (Mez) L.B.Sm. em vista frontal C: Secção transversal da lâmina foliar de Wittrockia superba Lindm. D: Detalhe da secção transversal da lâmina foliar de W. superba, evidenciando um canal de aeração. $\mathrm{Al}=$ Células da ala do tricoma; $\mathrm{Ca}=$ Canais de aeração; $\mathrm{D}=$ células do disco do tricoma; Setas indicam os cristais de sílica.

Características anatômicas relacionadas às suas flores também tornam a família um interessante alvo de estudo. A presença de nectários septais, tributo pouco comum na ordem Poales, é descrita para Bromeliaceae, sendo possíveis duas conformações: infraloculares, quando os ovários são súperos ou semi-ínferos e os nectários ocorrem abaixo dos lóculos; e septais interloculares, quando os ovários são ínferos e os nectários ocorrem nos septos das folhas carpelares, entre os lóculos do ovário (Sajo et al.2004a; Linder \& Rudall, 2005) (Fig. 
4A-B). Tais estruturas já foram estudadas em relação à composição química de seus produtos (Bernardello et al. 1991; Stahl et al., 2012), sua ultraestrutura e anatomia (Fiordi \& Palandri, 1982; Kulkarni \& Pai, 1982; Sajo et al. 2004a) e também sobre a sua posição na flor relacionando-a com a evolução da epiginia no grupo (Sajo et al. 2004a).

Outro aspecto amplamente estudado é a estrutura anatômica do óvulo em Bromeliaceae. Estudos descrevem que os óvulos podem apresentar estruturas características denominadas de apêndices calazais e micropilares (Sajo et al. 2004b; Fagundes \& Mariath, 2014; Nogueira et al. 2015; Kuhn et al. 2016;) (Fig.4C-D). Os apêndices calazais podem ter origem dérmica ou subdérmica e podem ser reconhecidos como um crescimento pronunciado na região da calaza (Fig. 4C-D). Já os apêndices micropilares podem ser reconhecidos pelo crescimento dos tegumentos (interno e externo), formando uma conspícula protrusão (Sajo et al. 2004b).

\subsection{Importância econômica e ecológica de Bromeliaceae}

Bromeliaceae é popularmente conhecida como a família do abacaxi. Mas sua relevância econômica vai muito além da comercialização dos saborosos frutos de Ananas comosus (L.) Merr. Devido à beleza de suas folhas e ao colorido de duas inflorescências, espécies de Bromeliaceae são utilizadas como plantas ornamentais (Reitz, 1983). A grande procura pelas Bromeliaceae de valor ornamental intensificou o extrativismo destas plantas de seu ambiente natural nos últimos anos, colocando alguns táxons em maior grau de ameaça. 

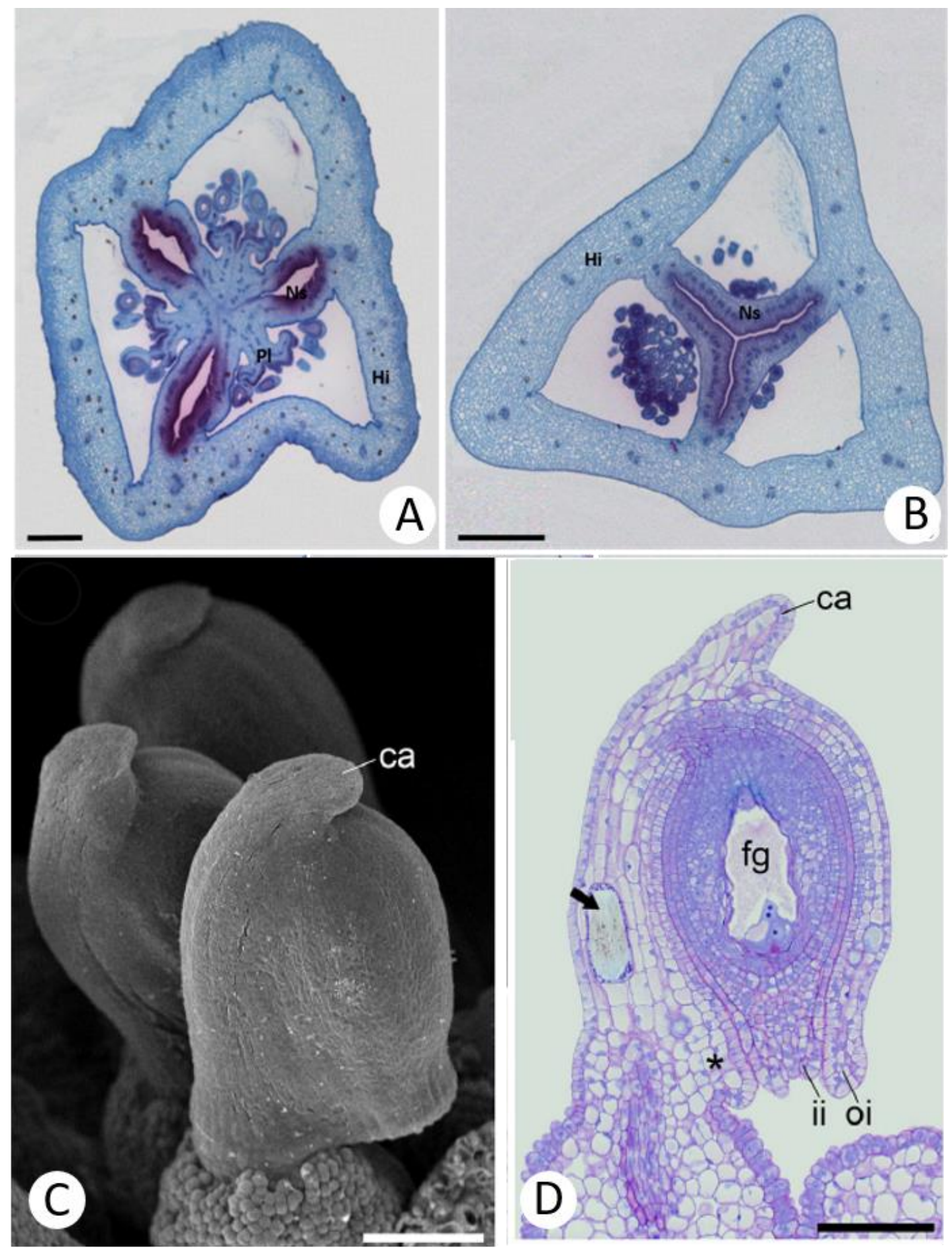

Figura 4: A-B Secção transversal do ovário evidenciando o nectário septal (Ns). A: Aechmea distichantha. Lem. B: Canistropsis billbergioides. (Schult \& Schult. f.) Leme C: Microscopia Eletrônica de Varredura do óvulo de Billbergia nutans H. Wendl. Ex. Regel evidenciado o apêndice calazal (Ca). D: Secção longitudinal do óvulo de $B$. nutans. ca= apêndice calazal, $\mathrm{fg}=$ megagametófito; oi= tegumento externo; ii= tegumento interno; $\mathrm{Hi}=$ hipoderme; $\mathrm{Ns}=\mathrm{Nectário} \mathrm{septal} ; \mathrm{Pl}=$ placenta. $\mathrm{A}$ e B: Retirado de Oliveira et al. (2016). C e D: Retirado de Fagundes \& Mariath (2014). 
Devido a água acumulada armazenada em seu tanque central (fitotelma), as Bromeliaceae têm importância ecológica fundamental, sendo um elemento importante para a ampliação da diversidade. (Oliveira, 2004). Numerosas espécies de animais utilizam essas plantas para a sua reprodução (como é o caso dos anfíbios), forrageamento e refúgio contra seus predadores (Rocha et al., 1997). Algumas espécies de plantas germinam e se desenvolvem no fitotema, possuindo um papel importante no estabelecimento de outras espécies vegetais, como por exemplo em ambientes de restinga. (Fialho \& Furtado, 1993; Zaluar \& Scarano, 2000).

Todavia, a importância ecológica não se restringe à água acumulada em suas rosetas. As flores das Bromeliaceae, por meio do néctar produzido, atraem uma gama de polinizadores que nelas obtêm recursos alimentares. Assim, durante o período de floração, essas plantas constituem fonte importantíssima de alimento para diversos animais, como abelhas, mariposas, beija-flores, vespas e borboletas. Suas flores também são reduto para diversas espécies de ácaros (Rocha et al., 1997).

\subsection{Estrutura geral da tese}

No primeiro capítulo, o trabalho aborda o estudo da anatomia em espécies pertencentes ao Complexo Nidularióide com enfoque filogenético. Este grupo é formado pelos gêneros Nidularium Lem., Neoregelia L.B.Sm., Edmundoa Leme e Wittrockia Lindm. (Leme, 1998). O interesse surgiu ao perceber que os gêneros pertencentes a este Complexo possuem difícil delimitação e, provavelmente, não são monofiléticos. No entanto, o Complexo como um todo emerge sempre como um grupo monofilético nas filogenias recentes de Bromelioideae. Para tanto, foram selecionadas espécies pertencentes ao Complexo Nidularióide abordadas por Silvestro et al. (2014), buscando estabelecer sinapomorfias anatômicas para o grupo. Neste sentido, este capítulo aborda aspectos morfoanatômicos foliares sob uma perspectiva filogenética. 
No segundo capítulo, selecionamos 16 espécies, pertencentes as subfamílias Bromelioideae, Pitcairnoideae e Tillandsoideae, para o estudo comparativo do androceu e gineceu. Nosso objetivo, neste trabalho, foi descrever caracteres que possam ser utilizados na sistemática do grupo, assim como discutí-los sob uma perspectiva ecológica e filogenética. Assim, descrevemos caracteres referentes a anatomia geral do androceu e ginegeu, caracteres referentes a vascularização destes verticilos e também referentes às estruturas secretoras. Adicionalmente, propusemos uma nova sinapomorfia anatômica, referente à vascularização do gineceu, para Pitcairnoideae.

No terceiro capítulo, ao observar o desenvolvimento foliar em espécies pertencentes ao gênero Tillandsia L. (Tillandsoideae), notamos a presença de tricomas glandulares próximos ao meristema apical caulinar. Assim, neste capítulo, nosso objetivo foi descrever estes tricomas glandulares e realizar testes histoquímicos pertinentes para a classificação dos principais compostos presentes no exudado destas estruturas. 


\section{Referências}

APG IV. 2016. An update of the Angiosperm phylogeny group classification for the orders and families of flowering plants: APG IV. Botanical Journal of Linnean Society, 181: 1-20.

Benzing, D.H. 1976. Bromeliad trichomes: structure, function and ecological significance. Selbyana. 1:330-348.

Benzing, D.H. 2000. Bromeliaceae: profile of an adaptative radiation. New York: Cambridge University Press.

Bernardello, L.M.; Galetto, L.; Juliani, H.R. 1991. Floral nectar, nectary structure and pollinators in some Argentinean Bromeliaceae. Annals of Botany 67 (5): 401-411.

BFG. 2015. Growing knowledge: an overview of Seed Plant diversity in Brazil. Rodriguésia, v.66, n.4, p.1085-1113. (DOI: 10.1590/2175-7860201566411)

Brown, G. K.; Gilmartin, A. J. 1989a. Stigma types in Bromeliaceae- a systematic survey. Systematic Botany. 14: 110-132.

Brown, G.K.; Gilmartin, A.J. 1989b. Cromosssomes number in Bromeliaceae. American Journal of Botany 76 (5):657-665.

Cronquist, A. 1981. An Integrated System of Classification of Flowering Plants. New York: Columbia University Press.

Dahlgren, R.M.T.; Clifford, H.T.; Yeo, P.F. 1985. The families of the monocotyledons: structure, evolution and taxonomy. Berlin: Springer-Verlag.

Escobedo-Sarti, J.; Ramírez, I.; Leopardi, C.; Carnevali, G.; Magallón, S.; Duno, R.; Mongradón, D. 2013. A phylogeny of Bromeliaceae (Poales, Monocotyledonaee) derived from an evaluation of nine methods. Journal of Systematics and Evolution. 51 (6): 743-757.

Fagundes, N.F.; Mariath, J.E.A. 2014. Ovule ontogeny in Billbergia nutans in the evolutionary contex of Bromeliaceae (Poales). Plant. Systematics and Evolution 300:1323-1336.

Fialho, R.F.; Furtado, A.L.S. 1993. Germination of Erythroxylum ovalifolium (Erythroxylaceae) seeds within the terrestrial bromeliad Neoregelia cruenta. Briotropica. 25:359-362. 
Fiordi, A.C.; Palandri, M.R. 1982. Anatomic and ultrastructural study of the septal nectary in some Tillandsia (Bromeliaceae) species. Caryologia 35(4): 477-489.

Gilmartin, A.J.; Brown, G.K. 1987. Bromeliales, Related monocots, and resolution of relationships among Bromeliaceae Subfamilies.Systematic Botany 12 (4): 493-500.

\section{Givnish, T.J.; Millam, K.C.; Evans, T.M.; Hall, J.C.; Pires, J.C.; Berry, P.E.; Sytsma, K.J.} 2004. Anciant vicariance or recent long distance dispersal? Inferences about phylogeny and south american-african disjunctions in Rapateaceae and Bromeliaceae based on $n d h \mathrm{~F}$ sequence data. International Plant Science. 4 (Supplem.), S35-S54.

Givnish, T.J.; Millan,K.C.; Berry, P.E.; Systma, K.J. 2007. Phylogeny, adaptive radiation, and historical biogeography of Bromeliaceae inferred from $n d h F$ sequence data. Aliso, $\mathrm{n}$. 23, p. 3-26.

Givnish, T.J.; Barfuss, M.H.J.; Van Ee, B.; Riina, R.; Schulte, K.; Horres, R.; Gonsiska, P.A.; Jabaily, R.S.; Crayn, D.M.; Smith, A.C.; Winter, K.; Brown, G.K.; Evans, T.M.; Holst, B.K.; Luther, H.; Till, W.; Zizka, G.; Berry, P.E.; Systma, K.J. 2011. Phylogeny, adaptive radiation, and historical biogeography in Bromeliaceae: insights from an eightlocus plastid phylogeny. American Journal of Botany, n.98, v.5, p 1-24.

Jacques-Felix, H. 2000. The discovery of a bromeliad in Africa: Pitcairnia feliciana. Selbyana $21(1,2): 118-124$.

Krauss, B.H. 1949. Anatomy of the vegetative organs of the pineapple Ananas comosus (L.) Merr.: II The leaf. Botanical Gazette. 110 (3): 333-404.

Kuhn, S.A.; Nogueira, F.M.; Fagundes, N.F.; Mariath, J.E. 2016. Morphoanatomy of the ovary and ovule in Bromeliaceae subfamily Tillandsoideae and its systematic relevance. Botanical Journal of Linnean Society 181:343-361.

Kulkarni, R.A.; Pai, R.M. 1982. The floral anatomy of Puya spathacea Mez. (Bromeliaceae) with special reference to nectaries. Proceedings of the Indian Academy of Science 91 (6): 473-478.

Leme, E.M.C. 1998. Canistropsis - Bromélias na Mata Atlântica. Rio de Janeiro: Salamandra

Linder, H.P.; Rudall, P.J. 2005. Evolutionary history of Poales. Annual Review of Ecology, Evolution and Systematics 36: 107-124. 
Luther, H. 2012. An alphabetical list of bromeliad binomials. 12th ed. The Bromeliad Society International. The Marie Selby Botanical Gardens, Sarasota \& Bromeliad Society International, $44 \mathrm{p}$.

Martinelli, G., Vieira, C.M., Gonzalez, M., Leitman, P., Piratininga, A., Costa, A.F.; Forzza, R.C. 2008. Bromeliaceae da Mata Atlântica brasileira: lista de espécies, distribuição e conservação. Rodriguésia, 59 (1): 209-258.

Mez, C. 1896. Bromeliaceae. In: Candolle, A. L.P.P. de et Candolle A.C.P.de (Eds.). Monographiae Phanerogamarum... Paris: G. Masson.

Nogueira, F.M.; Fagundes, N.F.; Kuhn, S.A.; Fregonezi, J.N.; Mariath, J.E.A. 2015. Ovary and ovule anatomy in the nidularioid complex and its taxonomic utility (Bromeliaceae: Bromelioideae). Botanical Journal of Linnean Society 177: 66-77.

Oliveira, R.R. 2004. Importância das bromélias epífitas na ciclagem de nutrientes na Floresta Atlântica. Acta Botânica Brasílica. 18(4): 793-799.

Oliveira, F.M.C.; Souza, A. M.; Corrêa, B. B. R.; Maeda, T. M.; Melo-de-Pinna, G. F. A. 2016. Anatomia floral de Aechmea distichantha Lem. e Canistropsis billbergioides (Schult. \& Schult. f.) Leme (Bromeliaceae). Hoehnea 43 (2), 183-193.

Prychid, C.J.; Rudall, P.; Gregory, M. 2004. Systematics and biology of silica bodies in Monocotyledons. The Botanical Review, 69: 377-440.

Reitz, R. 1983. Bromeliáceas e a malária - bromélia endêmica. Flora ilustrada Catarinense.

Rocha, C.F.D.; Cogliatti-Carvalho, L.; Almeida, D.R.; Freitas, A.F.N. 1997. Bromélias: ampliadoras da diversidade. Bromélia. 4: 7-10.

Sajo, M.G; Rudall, P.J.; Prychid, C.J. 2004. Floral anatomy of Bromeliaceae, with particular reference to the evolution of epigyny and septal nectaries in comelinid monocots. Plant Systematics and Evolution 247: 215-231.

Sajo, M.G.; Prychid, C.J.; Rudall, P.J. 2004b. Structure and development of the ovule in Bromeliaceae. Kew Bulletin. 59: 261-267.

Silvestro, D.; Zizka, G.; Schulte, K. 2014. Desentangling the effects of key innovations on thediversification of Bromelioideae (Bromeliaceae). Evolution 68 (1): 163-175. 
Smith L.B. \& Downs R.J. 1974. Pitcairnioideae (Bromeliaceae). Flora Neotropica Monograph 14: 1-662.

Smith L.B. \& Downs R.J. 1977. Tillandsioideae (Bromeliaceae). Flora Neotropica Monograph 14: 663-1492.

Smith L.B. \& Downs R.J. 1979. Bromelioideae (Bromeliaceae). Flora Neotropica Monograph 14: $1493-2142$.

Stahl, J.M.; Nepi, M.; Galetto, L.; Guimarães, E.; Machado, S.R. 2012. Functional aspects of floral néctar secretion os Ananas ananassoides, an ornithophilous bromeliad from the Brazilian savanna. Annals of Botany 109 (7): 1243-1252.

Tomlinson, P. 1969. Commelinales- Zingiberales. In: Metcalf, CR. (ed.) Anatomy of the Monocotyledons: III. Claredon Press, Oxford. Pp: 193-294.

Zaluar, H.L.T.; Scarano, F.R. 2000. Facilitação em restingas e moitas: um século de buscas por espécies focais. In: Esteves, F.A. \& Lacerda, L.D. (Eds.). Ecologia de restingas e lagoas costeiras do Brasil. Rio de Janeiro: NUPEM, UFRJ.

Wanderley, M.G.L.; Moreira, B.A. 2000. Notas taxonômicas sobre Nidularium Lem. e Wittrockia Lindm. (Bromelioideae, Bromeliaceae). Acta Botanica Brasilica, 14 (1): .1-9.

Wanderley, M.G.L.; Martins, S.E. 2007. Bromeliaceae. In: Wanderley, M.G.L.; Shepherd, G.J.; Melhem, T.S.; Giulietti, A. M. (Org.). Flora Fanerogâmica do Estado de São Paulo. São Paulo: Instituto de Botânica. 5: 1-476. 
Conclusões Gerais

$(16)$ 


\section{Conclusões Gerais}

A proposta deste trabalho foi estabelecer caracteres anatômicos que fossem úteis para a sistemática em Bromeliaceae, bem como discuti-los sob uma perspectiva filogenética.

No primeiro capítulo, o objetivo principal foi estabelecer sinapomorfias morfológicas e anatômicas para o clado Nidularióide. Com as reconstruções de estado de carácter, pudemos concluir que caracteres morfológicos frequentemente utilizados na delimitação dos gêneros pertencentes ao Complexo Nidularióide, tais como o tipo de inflorescência, presença de flores pediceladas/sésseis, a presença de apêndices nas pétalas, e a presença de calosidades nas pétalas são homoplásticos, e não deveriam ser utilizados na circunscrição destes gêneros. A maioria dos caracteres anatômicos analisados também representam homoplasias, no entanto, foi possível estabelecer duas novas sinapomorfias para o clado Nidularióide: (1) presença de tricomas peltados absorventes cujas células da ala são alongadas e (2) presença de espessamento na parede periclinal interna das células epidérmicas adaxiais da lâmina foliar.

Neste estudo, percebemos que trabalhos que envolvem evolução de caracteres em Bromelioideae ainda são escassos, principalmente os que envolvem a análise de caracteres anatômicos. Ainda, demostramos que a anatomia foliar em Bromelioideae pode fornecer subsídios para futuros estudos de evolução neste grupo, propondo novas sinapomorfias para este grupo que é morfologicamente tão diverso e de difícil delimitação genérica.

No segundo capítulo, ao estudar a anatomia floral de espécies pertencentes à três das oito subfamílias de Bromeliaceae, pudemos descrever caracteres que podem ser úteis na sistemática da família. Propusemos uma nova sinapomorfia anatômica para 
Pitcairnoideae, baseando-se no padrão de vascularização do gineceu. Também encontramos caracteres que possuem significância ecológica, tais como a presença de epiderme cônica, cujo formato é dado pela deposição da cutícula. Acreditamos que, pela ocorrência nas porções apicais dos filetes e estiletes, bem como nas anteras e estigmas, esta característica esteja envolvida com a atração de polinizadores. As estruturas secretoras também foram analisadas, estando presentes os nectários septais/ infraloculares, obturador e tecido transmissor. Em relação aos nectários, optamos por chamar de septais apenas os presentes em Bromelioideae, cujos ovários são ínferos e estes ocorrem verdadeiramente nos septos. Já para os nectários de Pitcairnoideae e Tillandsoideae, optamos por chamar de nectários infraloculares, uma vez que a porção secretora ocorre abaixo dos lóculos dos ovários.

Os caracteres descritos neste capítulo mostram a necessidade de estudos posteriores, envolvendo as oito subfamílias de Bromeliaceae, a fim de melhor entender a evolução floral neste grupo.

No terceiro capítulo, ao analisarmos três espécies do gênero Tillandsia L. (Tillandsoideae), descrevemos pela primeira vez, a presença de coléteres em Bromeliaceae. Estes coléteres são tricomas, que ocorrem na face adaxial da bainha foliar, próximos ao meristema apical caulinar. Possuem secreção de composição mista, i.e. secretam substâncias lipofílicas e hidrofílicas. Visto que a espécie em que estes coléteres ocorrem é epífita e não forma um tanque central com a sobreposição das bainhas foliares, esta secreção provavelmente tem relação com a proteção do meristema apical caulinar contra o dessecamento.

Acreditamos que estas estruturas ocorram em outros membros da família e que, devido à ausência de estudos de desenvolvimento foliar e floral, ainda não haviam sido documentados para Bromeliaceae, e possuem poucos registros na Monocotiledôneas em 
geral. Assim, reinforçamos a necessidade de futuros estudos envolvendo o desenvolvimento foliar em Bromeliaceae.

Ao analisar os resultados dos três capítulos conjuntamente, percebe-se que os caracteres anatômicos têm grande potencial para o uso na sistemática de Bromeliaceae, no entanto estes são subutilizados. Assim, acreditamos que os caracteres descritos nesta tese possuem relevância na sistemática do grupo e podem ser aplicados em futuros estudos de evolução em Bromeliaceae. 